\title{
Is Febuxostat Use Associated With Increased Risk of Cardiovascular Disease Events? The Answer Is Crystal Clear
}
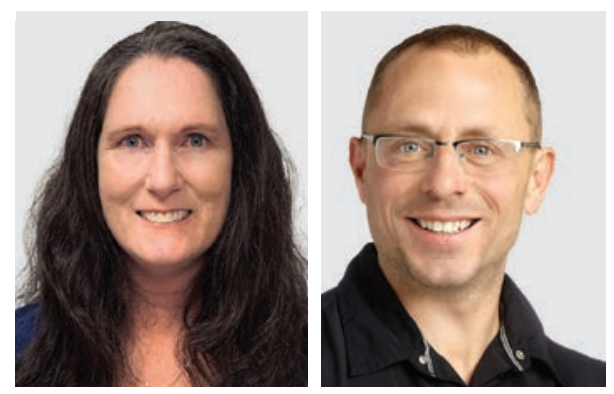

\author{
Alexandra Charlton ${ }^{1}$ and Paul Anthony MacMullan ${ }^{1}$
}

Gout is the most common inflammatory arthritis ${ }^{1}$, historically poorly managed, and is arguably the only one of the inflammatory arthritides that is curable with appropriate urate-lowering therapy $(\mathrm{ULT})^{2}$. Whereas febuxostat has been shown to be a more effective $\mathrm{ULT}^{3}$, allopurinol has been the cornerstone of treatment for decades; however, allopurinol is problematic in the elderly, those with renal impairment, and those who carry the HLA-B*5801 antigen who are primarily of Asian origin. Severe cutaneous allergic reactions (SCAR) are more common in the latter group as they carry up to a $30 \%$ mortality rate for the most severe form. Based on this, the Clinical Pharmacogenetics Implementation Consortium and other organizations have declared that allopurinol should be considered contraindicated in HLA-B*5801-positive individuals ${ }^{4}$. Even in those who do not carry the antigen, adverse skin reactions to allopurinol are also frequently seen; on the other hand, febuxostat carries a lower risk for these 5 . Including HLA-B*5801-positive patients, the elderly, those with renal impairment, and others who do not tolerate allopurinol at maximal doses, there is a significant proportion of patients for whom febuxostat is the best alternative. However, the use of febuxostat has faced significant challenges, including the "black box" warning from the FDA and the subsequent Health Canada statement ${ }^{6,7}$ regarding cardiovascular $(\mathrm{CV})$ risk and death, both of which have created a great deal of confusion for prescribers.

Febuxostat, even prior to any perceived adverse CV disease (CVD) risk profile, has been somewhat restricted in coverage by insurers across Canada, primarily due to the initial cost difference when compared to allopurinol. Several provincial plans

${ }^{1}$ A., Charlton, Clinical Pharmacist AHS, Doctor of Pharmacy, DPharm (PhD), P.A. MacMullan, MD, Clinical Associate Professor, Division Chief, Division of Rheumatology, Department of Medicine, Cumming School of Medicine, University of Calgary, Calgary, Alberta, Canada. Address correspondence to Prof. P.A. MacMullan, Division Chief of Rheumatology, University of Calgary, 2500 Hospital Drive, Calgary, $A B$ T2N 1N4, Canada.Email:paul.macmullan@ucalgary.ca. require "demonstrated hypersensitivity to allopurinol" prior to coverage of febuxostat, which seems unnecessary due to the inability to predict those who may have severe reactions. The province of Alberta also requires failure or contraindication to sulfinpyrazone, a second-line uricosuric agent that has very little evidence to support its use in gout ${ }^{8}$ and is associated with a high risk of bleeding due to interactions with antiinflammatories and anticoagulants 9 . Another widely used uricosuric, probenecid (which is not commercially available in Canada), has a better safety profile, but these agents are not as effective ${ }^{8}$ as those agents that interfere with urate production. In fact, uricosurics are not effective in patients with even mild chronic kidney disease and may result in further renal complications with development of nephrolithiasis ${ }^{10}$. Other uricosurics-lesinurad, benzbromarone, and the novel pegloticase-are not available in Canada and all can have significant side effects or have warnings and concerns associated with them ${ }^{11}$. Given the lack of efficacy and safety of these other drugs, it is unfortunate that recent publications have recommended against febuxostat based on the results of a single study $^{12}$. Canadians with gout who also have preexisting CVD would then be deprived of a more effective first-line option where allopurinol is contraindicated.

It is within this context that the recent article by Deng, et al, published in this issue of The Journal ${ }^{13}$ is of the utmost clinical importance for the various reasons. First, the background and aim of the study have been clearly defined. Second, notwithstanding the inherent problems associated with metaanalyses, the methodology and statistics are sound, the heterogeneity of the various studies is clearly evaluated, and any issues leading to potential bias are comprehensively addressed. Third, the quality of the evidence evaluated is appropriately graded in accordance with international best practice ${ }^{14}$. Finally, the finding that patients with gout-already at an inherently disproportionate risk of $\mathrm{CVD}^{15,16}$ - who receive febuxostat are not put at any further risk, is an important contribution to the literature.

The CARES randomized controlled trial $(\mathrm{RCT})^{17}$ showed

\section{See Febuxostat and mortality, page 1082}


an increase in both $\mathrm{CV}$ death and all-cause mortality with a number needed to harm (NNH) of 91 and 72 , respectively ${ }^{12}$, for febuxostat over allopurinol. This was the study that prompted the federal warnings in North America, but several recent metaanalyses ${ }^{18,19,20,21}$, including this one by Deng, et $\mathrm{l}^{13}$, have questioned the safety outcomes seen in this single RCT. Deng, et a $l^{13}$ provides valuable perspective and real-world insight by including an analysis of patients with gout who received a placebo, and also by including further studies reflecting a more diverse population than CARES. As the authors correctly detail, restricting the use of an effective drug, especially in patients who cannot or should not take allopurinol, may also lead to negative outcomes that were not accounted for in the CARES study. Further, only $3 \%$ of patients in the CARES study were of Asian descent and thus, those at greater risk of SCAR with allopurinol were not adequately represented. In those at high risk of allopurinol hypersensitivity, a slight cardiac benefit may not be enough to overcome the morbidity and mortality risks associated with SCAR. This is consistent with a previous subanalysis of the Asian subjects in the trial that found febuxostat to be safer and more effective than allopurinol ${ }^{21}$.

What this study by Deng, et al best conveys is that most gout patients are at risk for CV death and all-cause mortality ${ }^{13}$. We know that gout and hyperuricemia are strongly associated with CVD and increased CV mortality ${ }^{15,16}$. Additionally, those with gout frequently have other comorbidities associated with CVD and increased mortality such as obesity, diabetes/ metabolic syndrome, hypertension, and hypercholesterolemia. By including a more diverse population for analysis and through evaluation of those subjects receiving a placebo, we see that there is no increased CV risk associated with febuxostat over patients who receive no treatment or over those who receive less effective second-line treatments. The morbidity associated with inadequately treating gout, which is essentially a curable disease, should also not be discounted in this discussion and the optimal treatment of this most common inflammatory arthritis should be a primary goal. The metaanalysis by Deng, et al ${ }^{13}$ provides muchneeded clarity for prescribers regarding the use of febuxostat in their gout patients with comorbid CVD and with an intolerance or contraindication to allopurinol.

\section{REFERENCES}

1. Richette P, Doherty M, Pascual E, Barskova V, Becce F, Castaneda J, et al. 2018 updated European League Against Rheumatism evidence-based recommendations for the diagnosis of gout. Ann Rheum Dis 2020;79:31-8.

2. Bernal JA, Quilis N, Andrés M, Sivera F, Pascual E. Gout: optimizing treatment to achieve a disease cure. Ther Adv Chronic Dis 2016;7:135-44.

3. Becker MA, Schumacher HR, Espinoza LR, Wells AF, MacDonald P, Lloyd E, et al. The urate-lowering efficacy and safety of febuxostat in the treatment of the hyperuricemia of gout: the CONFIRMS trial. Arthritis Res Ther 2010;12:R63.

4. Hershfield MS, Callaghan JT, Tassaneeyakul W, Mushiroda T, Thorn CF, Klein TE, et al. Clinical Pharmacogenetics Implementation Consortium guidelines for human leukocyte antigen-B genotype and allopurinol dosing. Clin Pharmacol Ther 2013;93:153-8.
5. Yu KH, Lai JH, Hsu PN, Chen DY, Chen CJ, Lin HY. Safety and efficacy of oral febuxostat for treatment of HLA-B*5801-negative gout: a randomized, open-label, multicentre, allopurinol-controlled study. Scand J Rheumatol 2016;45:304-11.

6. U.S. Food and Drug Administration. FDA adds boxed warning for increased risk of death with gout medicine uloric (febuxostat). [Internet. Accessed October 5, 2020.] Available from: www.fda.gov/ drugs/fda-drug-safety-podcasts/fda-adds-boxed-warning-increasedrisk-death-gout-medicine-uloric-febuxostat

7. Health Canada. Uloric (febuxostat) - increased risk of cardiovascular fatal outcomes. [Internet. Accessed October 5, 2020] Available from: healthycanadians.gc.ca/recall-alert-rappel-avis/ hc-sc/2019/71511a-eng.php

8. Kydd ASR, Seth R, Buchbinder R, Edwards CJ, Bombardier C. Uricosuric medications for chronic gout. Cochrane Database Syst Rev 2014;11:CD010457.

9. Dabigatran-Ibuprofen-ASA-Ibuprofen. Interactions. Lexicomp. Wolters Kluwer Health, Inc. Riverwoods, IL. [Internet. Accessed September 28, 2020.] Available from: online.lexi.com

10. Perez-Ruiz F, Hernandez-Baldezon S, Herrero-Beites AM, Gonzalez-Gay MA. Risk factors associated with renal lithiasis during uricosuric treatment of hyperuricemia in patients with Gout. Arthitis Care Res 2010;62;1299-305.

11. Strilchuk L, Fogacci F, Cicero F. Safety and tolerability of available urate-lowering drugs: a critical review. Expert Opin Drug Saf 2019;18:261-71.

12. Ton J, Kolber N. Who let the gout out? Targeting uric acid levels in treating gout tools for practice. [Internet. Accessed October 5 , 2020.] Available from: gomainpro.ca/wp-content/uploads/ tools-for-practice/1589571963_tfp262allopurinolfv.pdf

13. Deng H, Zhang BL, Tong JD, Yang XH, Jin HM. Febuxostat use and risks of cardiovascular $(\mathrm{CV})$ events, $\mathrm{CV}$ death, and all-cause mortality: a metaanalysis of randomized controlled trials. J Rheumatol 2021;48:1082-9.

14. BMJ Practice. What is GRADE? [Internet. Accessed October 5, 2020] Available from: bestpractice.bmj.com/info/toolkit/ learn-ebm/what-is-grade

15. Hansildaar R, Vedder D, Baniaamam M, Tausche A-K, Gerritsen M, Nurmohamed MT. Cardiovascular risk in inflammatory arthritis: rheumatoid arthritis and gout. Lancet Rheumatol 2020 Sep 1 (E-pub ahead of print).

16. Lottmann K, Chen X, Schädlich PK. Association between gout and all-cause as well as cardiovascular mortality: a systematic review. Curr Rheumatol Rep 2012; 14:195-203.

17. White WB, Saag KG, Becker MA, Borer JS, Gorelick PB, Whelton $\mathrm{A}$, et al; CARES Investigators. Cardiovascular safety of febuxostat or allopurinol in patients with gout. N Engl J Med 2018;378:1200-10.

18. Cuenca JA, Balda J, Palacio A, Young L, Pillinger MH, Tamariz L. Febuxostat and cardiovascular events: a systematic review and meta-analysis. Int J Rheumatol 2019;2019:1076189.

19. Zhao L, Cao L, Zhao TY, Yang X, Zhu XX, Zou HJ, et al. Cardiovascular events in hyperuricemia population and a cardiovascular benefit-risk assessment of urate-lowering therapies: a systematic review and meta-analysis. Chin Med J 2020;133:982-93.

20. Al-Abdouh A, Khan SU, Barbarawi M, Upadhrasta S, Munira S, Bizanti A, et al. Effects of febuxostat on mortality and cardiovascular outcomes. Mayo Clin Proc Innov Qual Outcomes 2020;4:434-42.

21. Liu CW, Chang WC, Lee CC, Shau WY, Hsu FS, Wang ML, et al. The net clinical benefits of febuxostat versus allopurinol in patients with gout or asymptomatic hyperuricemia - a systematic review and meta-analysis. Nutr Metab Cardiovasc Dis 2019;29:1011-22. 